\title{
Quasiparticle tunneling in the lowest Landau level
}

\section{Journal Article}

\section{Author(s):}

Hennel, Szymon; Scheidegger, Patrick; Kellermeier, Max; Hofmann, Andrea; Krähenmann, Tobias; Reichl, Christian; Wegscheider, Werner (D); Ihn, Thomas Markus (D); Ensslin, Klaus (D)

Publication date:

2018-06-15

\section{Permanent link:}

https://doi.org/10.3929/ethz-b-000271709

\section{Rights / license:}

In Copyright - Non-Commercial Use Permitted

\section{Originally published in:}

Physical Review B 97(24), https://doi.org/10.1103/PhysRevB.97.245305 


\title{
Quasiparticle tunneling in the lowest Landau level
}

\author{
Szymon Hennel, ${ }^{*}$ Patrick Scheidegger, Max Kellermeier, Andrea Hofmann, Tobias Krähenmann, Christian Reichl, \\ Werner Wegscheider, Thomas Ihn, and Klaus Ensslin \\ Solid State Physics Laboratory, ETH Zürich, 8093 Zürich, Switzerland
}

(Received 20 April 2018; revised manuscript received 22 May 2018; published 11 June 2018)

\begin{abstract}
We measure quasiparticle tunneling across a constriction in the first Landau level. In the limit of weak backscattering, the dependence of the tunneling conductance on temperature and dc-bias is in qualitative disagreement with existing theories. For stronger backscattering, data obtained in the $v=1 / 3$ state can be fitted to weak backscattering theory with the predicted effective fractional charge of $e^{*}=e / 3$. The scaling parameter $g$ is however not universal and depends strongly on the gate voltage applied to the constriction. At $v=4 / 3$, a more complex picture emerges. We propose an interpretation in terms of selective tunneling between the multiple modes present at the edge.
\end{abstract}

DOI: 10.1103/PhysRevB.97.245305

\section{INTRODUCTION}

The edges of fractional quantum Hall $(\mathrm{FQH})$ fluids are predicted to form strongly interacting chiral one-dimensional systems [1]. From an experimental point of view, the hallmark of non-Fermi-liquid behavior at the FQH edge is observed in transport through a constriction in a two-dimensional electron gas: The tunneling conductance between two edge states brought into proximity shows a power-law dependence on temperature and dc bias [2]. Within a hydrodynamic formulation of the chiral Luttinger liquid, Wen derived a model of this tunneling conductance in which the relevant scaling parameters are the effective charge $e^{*}$ of the current-carrying quasiparticles (QPs) and an interaction parameter $g$ [3-5]. Both parameters can be extracted from experimental data by a nonlinear regression analysis, and both can be theoretically predicted for a given wave function, making tunneling conductance a potentially powerful probe of the nature of FQH states. This technique has indeed been used as a probe of fractional charge of complex states in the second Landau level, in particular at $v=5 / 2$ with limited success [6-9]. However, to date it has not been verified that the expected scaling parameters are obtained for states of which the wave function and the effective charge is known with a high degree of confidence, such as the Laughlin state at $v=1 / 3$ [10-13]. Experimental studies of QP tunneling between edge modes of Laughlin states have yielded unexpected results [14-19], and a quantitative analysis based on a fit to Wen's model has not been reported to date. Interpretations of the scaling parameters measured in the second Landau level, be it in terms of dependence on constriction geometry or competition between possible ground states, thus lack a solid experimental basis. In this paper we set out to verify quantitatively whether QP tunneling theory appropriately describes tunneling between edge channels at $v=1 / 3$ and $v=1+1 / 3$. We find that data in the weak backscattering regime is in qualitative contrast to theoretical

*hennels@phys.ethz.ch predictions. On the other hand, the theory developed for weak backscattering describes well experimental data acquired in the strong backscattering regime, and the analysis leads to an effective charge of $e / 3$. In this paper we focus on the presentation of experimental data, investigate the validity of the existing theory in different backscattering regimes, and discuss where a fitting analysis based on the existing theory might be useful to extract pertinent information. Understanding the quantum Hall edge and backscattering between fractional states is crucial to proceed with the study of interferometers and of other geometries designed to probe the proposed topological properties [20].

\section{EXPERIMENTAL SETUP}

We report data acquired in two different samples processed out of the same wafer, a single-side doped GaAs/AlGaAs heterostructure which hosts a 2 DEG residing $310 \mathrm{~nm}$ beneath the wafer's surface, with density $n_{\mathrm{s}} \approx 1.0 \times 10^{11} \mathrm{~cm}^{-2}$ and mobility $\mu \approx 4 \times 10^{6} \mathrm{~cm}^{2} \mathrm{~V}^{-1} \mathrm{~s}^{-1}$ at $4.2 \mathrm{~K}$. Both samples consist of a photolithographically defined $400 \mu \mathrm{m}$ wide Hall bar (schematically depicted in Fig. 1) on which several quantum point contacts (QPCs) were fabricated by electron-beam lithography and subsequent Ti/Au evaporation. The first sample features a row of independent QPCs. For the measurements we present here, only a single QPC with wide leads was defined by application of a negative voltage to the corresponding gates. The gap between the split gates was $2800 \mathrm{~nm}$. This QPC will be referred to in the following as "QPC 1" and can be seen on the left side of Fig. 1(b). On the second sample, a single QPC with a gap of $2000 \mathrm{~nm}$ and narrow gates [same design as on the right side of Fig. 1(b)] was defined and will be referred to as "QPC 2." Both QPCs were designed to be wide enough for the electronic density in their middle to be close to the density in the bulk 2DEG in the limit of low gate voltages.

Both samples have been measured in dilution refrigerators, using standard lock-in techniques at a frequency of $28 \mathrm{~Hz}$ to perform four-terminal measurements of differential resistance. ac and dc currents were supplied through $1 \mathrm{G} \Omega$ resistors in 


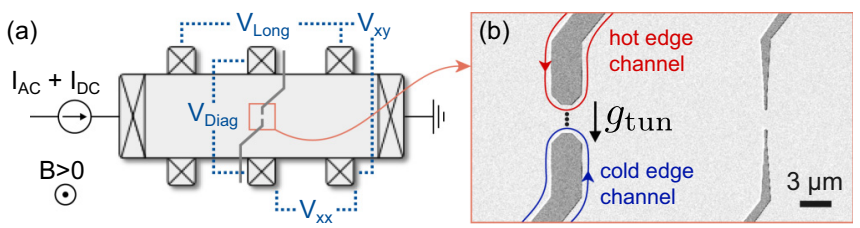

FIG. 1. (a) Schematic of the sample. Crosses represent Ohmic contacts to the Hall bar, voltage probes are indicated by dotted lines. (b) SEM image showing typical QPC geometries. The QPC on the left side has been used to acquire the data shown in Figs. 2, 3, and 4. The QPC on the right side has a similar design as the one used to acquire the data shown in Figs. 5 and 6. The tunneling conductance $g_{\text {tun }}$ between two counterpropagating edge modes in the QPC is represented schematically.

series with source and sample. We have verified that the $2 \mathrm{DEG}$ is fully depleted under the gates for all values of gate voltages that we report in this work.

A precise knowledge of the electronic temperature is crucial for the interpretation of our results. The sample hosting QPC 1 was in direct contact with the ${ }^{3} \mathrm{He} /{ }^{4} \mathrm{He}$ mixture in the mixing chamber of a refrigerator with a base temperature of $71 \mathrm{mK}$. The electronic temperature is therefore assumed to be equal to the mixing chamber temperature which was measured with a RuOx thermometer. The sample hosting QPC 2 was placed on a silver cold finger thermally anchored to a mixing chamber with a base temperature of $4.6 \mathrm{mK}$. The mixing chamber temperature was measured using an MFFT-1 noise thermometer [21]. The electronic temperature achieved for a given mixing chamber temperature is inferred from a calibration curve which we obtained from a previous study of activated behavior in fragile fractional quantum Hall states performed in the same dilution refrigerator.

\section{RESULTS AND DISCUSSION}

\section{A. Theoretical basis for our work}

We define $g_{\text {tun }}$ as the backwards tunneling conductance between the hot and the cold edge within the QPC, as shown in Fig. 1(b). Following previous works [6-9], we compute $g_{\text {tun }}$ according to

$$
g_{\text {tun }}=\frac{R_{\text {Diag }}-R_{\mathrm{xy}}}{R_{\mathrm{xy}}^{2}},
$$

where $R_{\text {Diag }}$ and $R_{\text {xy }}$ are the four-terminal resistances defined in Fig. 1. For the analysis of our data, we use the result obtained perturbatively within chiral Luttinger liquid theory for the differential tunneling conductance [22]

$$
g_{\text {tun }}\left(T, I_{\mathrm{dc}}, g, e^{*}\right)=A T^{2 g-2} F\left(g, \frac{e^{*} I_{\mathrm{dc}} R_{\mathrm{xy}}}{k_{\mathrm{B}} T}\right),
$$

where $T$ is the electronic temperature, the constant $A$ absorbs physical parameters not accessible experimentally,

$$
\begin{aligned}
F(g, x)= & B\left(g+i \frac{x}{2 \pi}, g-i \frac{x}{2 \pi}\right) \\
& \times\left(\pi \cosh \left[\frac{x}{2}\right]-2 \sinh \left[\frac{x}{2}\right] \Im\left(\Psi\left[g+i \frac{x}{2 \pi}\right]\right)\right),
\end{aligned}
$$
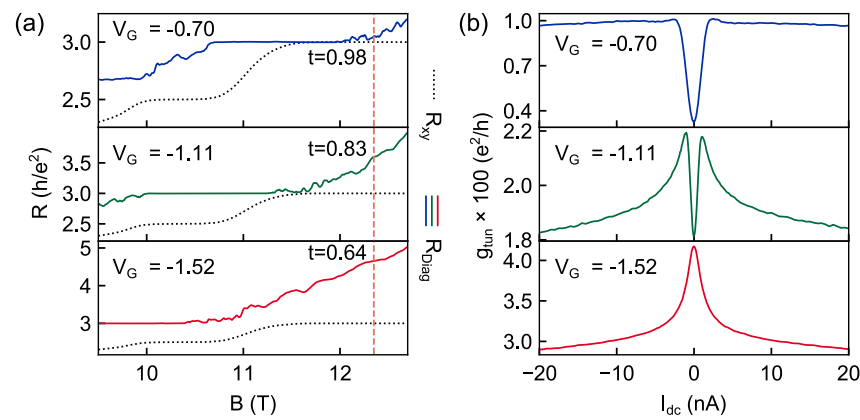

FIG. 2. (a) Diagonal resistance measured across QPC 1 (solid colored lines) for three different values of gate voltage. The black dotted line shows $R_{\mathrm{xy}}$ in the bulk of the sample, which is in the $v=1 / 3$ state at $B=12.35 \mathrm{~T}$ (value marked by the vertical dashed orange line). Labels indicate gate voltages $V_{\mathrm{G}}$ and the transmission $t$ of the $v=1 / 3$ edge channel at $B=12.35$ inferred from $R_{\text {Diag }}$. No dc bias was applied in this measurement. (b) dc-bias dependence of the tunneling conductance $g_{\text {tun }}$ across QPC 1 at $B=12.35$ for the same three values of gate voltage as in (a). All data shown in this figure have been acquired at an electronic temperature of $71 \mathrm{mK}$.

$$
\begin{aligned}
B(a, b) & =\frac{\Gamma(a) \Gamma(b)}{\Gamma(a+b)} \text { and } \\
\Psi(z) & \left.=\frac{\frac{d}{d z} \Gamma(z)}{\Gamma(z)} \quad \text { ( } \Gamma \text { is the gamma function }\right) .
\end{aligned}
$$

This perturbative result is expected to be valid for any $\mathrm{FQH}$ state in the limit of weak backscattering, i.e., a QPC transmission close to 1 . At filling factor $v=1 / 3$, the predicted scaling parameters [3] are $g=1 / 3$ and $e^{*}=e / 3$.

For Laughlin states at $v=1 / \mathrm{m}$, a nonperturbative solution for the tunneling conductance valid for any backscattering strength has been obtained by Fendley et al. [23,24]. The main result (Eq. (5.2) in Ref. [23]) is not expressed in closed form, making its application in a curve-fitting analysis of experimental data more challenging than for Eq. (2).

\section{B. Tunneling conductance at $v=1 / 3$}

The gate voltage dependence of the transmission of the $v=1 / 3$ edge state through QPC 1 is summarized in Fig. 2(a). For low values of gate voltage $\left(V_{\mathrm{G}}=-0.7 \mathrm{~V}\right)$, a clear $\nu_{\mathrm{QPC}}=$ $1 / 3$ plateau appears in $R_{\text {Diag }}$ and overlaps partially with the $v=1 / 3$ plateau in the Hall voltage $R_{\mathrm{xy}}$. The charge carrier density within the QPC $n_{\mathrm{QPC}}=9.2 \times 10^{10} \mathrm{~cm}^{-2}$ is indeed close to the bulk density $n_{\text {bulk }}=9.9 \times 10^{10} \mathrm{~cm}^{-2}$ (both those values have been obtained from the position of integer quantum Hall plateaus at low magnetic fields, data not shown). At $B=12.35 \mathrm{~T}$, near the middle of the plateau in $R_{\mathrm{xy}}$, the transmission of the $v=1 / 3$ mode is $t=\left(3 h / e^{2}\right) / R_{\text {Diag }} \approx 0.98$. In this regime, backscattering is expected to be weak, i.e., mediated by tunneling of QPs across the $v=1 / 3 \mathrm{FQH}$ fluid within the QPC, and the perturbative result in Eq. (2) is expected to hold. As gate voltage is made more negative, thus narrowing the QPC, the density within the QPC is reduced to $n_{\mathrm{QPC}}=8.7 \times 10^{10} \mathrm{~cm}^{-2}$. Correspondingly, the $v_{\mathrm{QPC}}=1 / 3$ plateau shifts to lower magnetic fields and the transmission of the $v=1 / 3$ mode is reduced to $t=0.83$. Finally, for highly negative gate voltages $\left(V_{\mathrm{G}}=-1.52 \mathrm{~V}\right)$, the density within the 

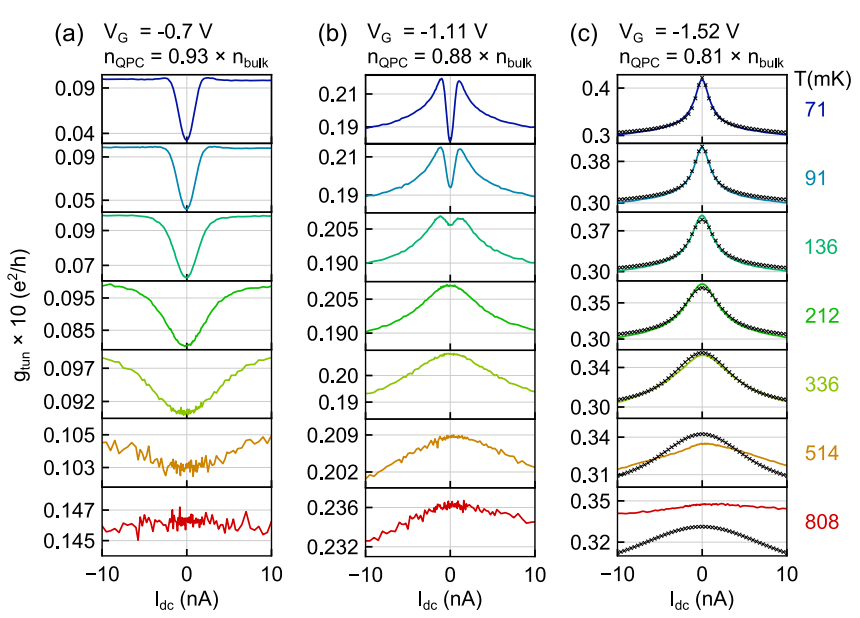

FIG. 3. (a) to (c) Temperature and dc-bias dependence of the tunneling conductance across QPC 1 . The gate voltage and the electronic density extracted from low-field data are indicated on top of each graph. The data acquired at $71 \mathrm{mK}$ are the same as shown in Fig. 2(b). Note that the seven $y$ axes in each column are independent, and that their scales are adapted for an optimal readability of the data. In (c), the black crosses follow the best fit to weak QP tunneling theory. Electronic temperatures are indicated on the right-hand side and are color coded for the convenience of the reader.

QPC is $n_{\mathrm{QPC}}=8.0 \times 10^{10} \mathrm{~cm}^{-2}$ and the transmission of the $v=1 / 3$ mode falls to $t=0.64$. Here, we expect backscattering to be in the strong regime, i.e., mediated by tunneling of single electrons across a compressible state in the QPC.

We have measured the bias dependence of the tunneling conductance $g_{\text {tun }}$ across QPC 1 using an ac current excitation of $I_{\mathrm{ac}}=100 \mathrm{pA}$. Decreasing the ac current excitation did not narrow the $g_{\text {tun }}$ peak within noise level. The results are summarized in Fig. 2(b). Surprisingly, in the range of gate voltages where backscattering is weak (around $-0.7 \mathrm{~V}$ ), the dc-bias dependence of $g_{\text {tun }}$ shows a minimum at zero bias, in striking contradiction to the zero-bias maximum predicted by QP tunneling theory [3,22]. Furthermore, a zero-bias peak is observed for highly negative values of gate voltage $(-1.52 \mathrm{~V})$, for which the transmission is below the threshold where we expect QP tunneling to occur. For intermediate gate voltages, we observe a $g_{\text {tun }}\left(I_{\mathrm{dc}}\right)$ curve which is the sum of a narrow zerobias minimum and a broader zero-bias maximum, indicating that the mechanisms present at low gate voltage and at high gate voltage coexist.

The amplitudes of all features in the bias dependence of $g_{\text {tun }}$ decrease with increasing temperature (see Fig. 3). Above $514 \mathrm{mK}$, we observe an overall increase in $g_{\text {tun }}$, which we attribute to thermal activation of the FQH liquid within the QPC because we also see an increase in the bulk $R_{\mathrm{xx}}$ at those temperatures. In the weak backscattering regime at $V_{\mathrm{G}}=$ $-0.7 \mathrm{~V}$, the zero-bias minimum evolves into a weak maximum at the highest temperature. In the intermediate regime at $V_{\mathrm{G}}=$ $-1.11 \mathrm{~T}$, the narrow minimum disappears below $212 \mathrm{mK}$ while the wide maximum is still visible at $514 \mathrm{mK}$. This is a further confirmation that the shape of the curve $g_{\text {tun }}\left(I_{\mathrm{dc}}\right)$ at low temperatures is the result of two coexisting mechanisms governed by different energy scales.
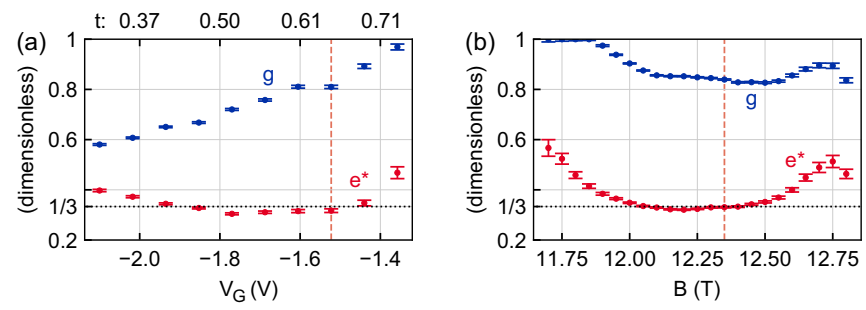

FIG. 4. Best-fit parameters $e^{*}$ and $g$ obtained in a least-square fit of Eq. (2) to the temperature and dc-bias dependence of the tunneling conductance across QPC 1. (a) Gate voltage dependence of the best-fit parameters for a fixed value of magnetic field $B=12.35 \mathrm{~T}$ [vertical dashed line in (b)]. The top axis shows the transmission $t$ of the $v=1 / 3$ mode at zero dc bias. The underlying data have been acquired in the same measurement as those displayed in Figs. 2 and 3. Data acquired for values of gate voltage less negative than $-1.35 \mathrm{~V}$ cannot be fit to QP tunneling theory. (b) Magnetic field dependence of the best fit parameters for a fixed value of gate voltage $V_{\mathrm{G}}=1.52 \mathrm{~V}$ [vertical dashed line in (a)]. The underlying data have been acquired under the same experimental conditions as for the results shown in (a), in a temperature range of $96 \mathrm{mK}$ to $411 \mathrm{mK}$.

Even though we do not expect QP tunneling to be the dominant contribution to backscattering at $V_{\mathrm{G}}=-1.52 \mathrm{~V}$, we are able to fit the model in Eq. (2) to the measured $g_{\text {tun }}$, with the two independent variables $T$ and $I_{\mathrm{dc}}$. The parameters of the best fit [indicated by black crosses in Fig. 3(c)] are $e^{*}=(0.32 \pm 0.01) e$ and $g=(0.81 \pm 0.01)$.

The data shown in Fig. 3 is part of a measurement series spanning the gate voltages $-0.4 \mathrm{~V}$ to $-2.1 \mathrm{~V}$ in steps of $82.5 \mathrm{mV}$. We observe zero-bias peaks in $g_{\text {tun }}$ for gate voltages more negative than $-1.275 \mathrm{~V}$. Equation (2) could be accurately fit to all $g_{\text {tun }}\left(T, I_{\mathrm{dc}}\right)$ datasets featuring a zero-bias maximum. The full gate voltage dependence of the best fit parameters $e^{*}$ and $g$ is shown in Fig. 4(a). The measured $e^{*}$ is close to the expected value of $e / 3$ in a wide range of gate voltage, but the interaction parameter $g$ is higher than the expected $g=1 / 3$ and its value is not universal but decreases as the constriction is made narrower by a more negative gate voltage. We have further measured $g_{\text {tun }}$ at fixed $V_{\mathrm{G}}=-1.52 \mathrm{~V}$ for different values of magnetic field. As shown in Fig. 4(b), the best fit parameters do not depend on magnetic field within the range of magnetic field corresponding to the $v=1 / 3$ plateau in the bulk of the sample.

Finally, we note that the best fit value of $e^{*}$ slightly increases for gate voltages more negative than $V_{\mathrm{G}}=-1.8 \mathrm{~V}$ (at this gate voltage the transmission of the $v=1 / 3$ channel is $t=0.50$ ) and is significantly higher than $e / 3$ for $V_{\mathrm{G}}<-2.0 \mathrm{~V}$ (where $t<0.37$ ). This behavior is qualitatively consistent with the results reported in experiments based on the analysis of shot noise in quantum point contacts $[25,26]$. The effective charge extracted from shot noise follows a universal dependence on channel transmission, with $e^{*}=e / 3$ in the limit of high channel transmission $(t>0.8)$, and a continuous increase towards $e^{*}=e$ in the limit of low transmission $[25,26]$. The increase of the measured effective charge with decreasing channel transmission is steeper in shot noise experiments than in our results: For $t=0.5$, the effective charge extracted from 

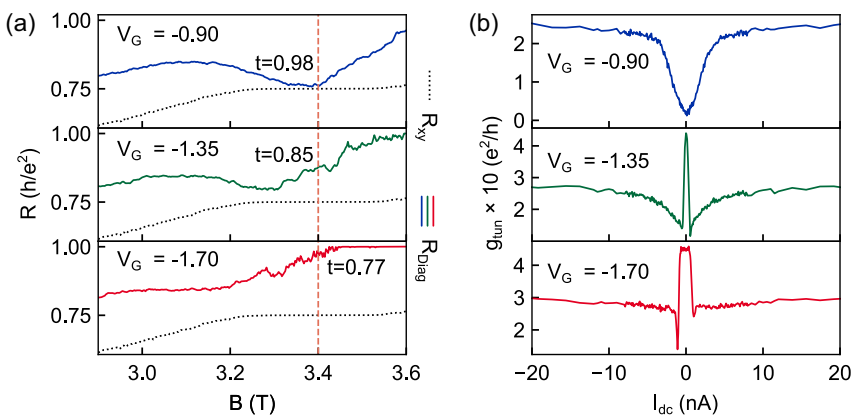

FIG. 5. (a) Diagonal resistance measured across QPC 2 (solid colored lines) for three different values of gate voltage. The black dotted line shows $R_{\mathrm{xy}}$ in the bulk of the sample, which is in the $v=4 / 3$ state at $B=3.4 \mathrm{~T}$ (value marked by the dashed orange line). Labels indicate gate voltages $V_{\mathrm{G}}$ and the transmission $t$ of the $v=4 / 3$ edge channels at $B=3.4 \mathrm{~T}$ inferred from $R_{\text {Diag. }}$ (b) dc-bias dependence of the tunneling conductance $g_{\text {tun }}$ across QPC 2 for the same three values of gate voltage as in (a). All data shown in this figure have been acquired at an electronic temperature of $9.6 \mathrm{mK}$.

shot noise is $e^{*} \approx 0.4$, while in our data $e^{*}=0.4$ is observed at a lower transmission of $t=0.3$.

\section{Tunneling conductance at $v=4 / 3$}

In order to test how other fractional states behave with respect to theory we have repeated our measurements at $v=2 / 3$ and $v=4 / 3$. At $v=2 / 3$, the situation is enriched by effects of dynamic nuclear spin polarization [27], prohibiting a similar analysis as we have shown for $v=1 / 3$. We therefore focus on the situation at filling factor $v=4 / 3$. The behavior of the tunneling conductance in the low backscattering limit is similar to the $v=1 / 3$ case, but a different picture emerges as the strength of backscattering is increased. All measurements of the tunneling conductance at $v=4 / 3$ were performed with an ac excitation of $I_{\mathrm{ac}}=20 \mathrm{pA}$. With $V_{\mathrm{G}}=-0.9 \mathrm{~V}$ applied to QPC 2 [see Fig. 5(a)] the electronic density in the QPC is approximately equal to bulk density and a high transmission of $t=0.98$ is observed at $B=3.4 \mathrm{~T}$, the field corresponding to the center of the $v=4 / 3$ plateau in the bulk $R_{\mathrm{xy}}$. In this regime, we expect to observe weak backscattering but find that the tunneling conductance shows a minimum at zero bias [see Fig. 5(b)]. In the case of strong backscattering $\left(V_{\mathrm{G}}=-1.7 \mathrm{~V}\right)$ a plateau emerges at $R_{\text {Diag }}=h / e$. This observation can be explained assuming that the $v=4 / 3$ edge state supports an inner $v=1$ mode and an outer $v=1 / 3$ mode. When $R_{\text {Diag }}$ is quantized to the value $h / e$, the $v=1 / 3$ mode is fully backscattered while the $v=1$ mode is still perfectly transmitted. The tunneling conductance calculated according to Eq. (1) is then exactly $g_{\text {tun }}=4 / 9 \times e^{2} / h \approx 0.44 e^{2} / h$.

For intermediate values of gate voltage $(-1.35 \mathrm{~V})$, the bias dependence of $g_{\text {tun }}$ features a narrow maximum superposed with a wide minimum. The resulting curve shape is reminiscent of the maximum with undershoots characteristic of Eq. (2) for $g<0.5$. However, as the tunneling conductance doesn't exceed the value $g_{\text {tun }}=4 / 9 e^{2} / h$ [dashed line in Fig. 6(a)], its temperature dependence is incompatible with the power-law behavior of Eq. (2). Noting that the $v=1$ mode is always fully transmitted and thus doesn't contribute to the tunneling
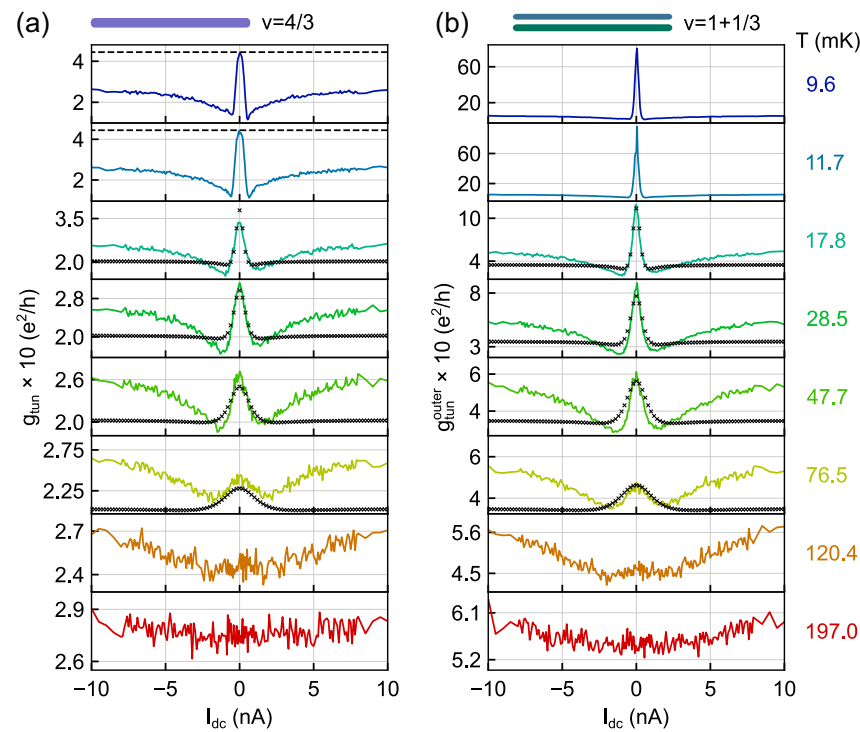

FIG. 6. Temperature and dc-bias dependence of the tunneling conductance across QPC 2 with a gate voltage of $-1.35 \mathrm{mV}$. (a) Tunneling conductance calculated from raw data following Eq. (1). The data obtained at $9.6 \mathrm{mK}$ (uppermost) are the same as shown in Fig. 5(b). Dashed lines in the two uppermost graphs mark the value $g_{\text {tun }}=4 / 9 \approx 0.44$. (b) Tunneling conductance of the outermost $v=1 / 3$ mode calculated according to Eq. (3) on the basis of the same data as in (a). The edge structure model corresponding to the method chosen for calculating $g_{\text {tun }}$ is symbolically represented on top of the figure. Note that the eight $y$ axes in each column are independent, and that their scales are adapted for an optimal readability of the data. In (a) and (b), the black crosses follow QP tunneling theory with $g=1 / 3, e^{*}=e / 3$, and manually adjusted amplitude and offset. Electronic temperatures are indicated on the left-hand side and are color coded for the convenience of the reader.

conductance, we define a quantity $g_{\text {tun }}^{\text {outer }}$ describing tunneling of the outer $v=1 / 3$ mode only:

$$
g_{\text {tun }}^{\text {outer }}=\frac{R_{\text {Diag }}^{\text {outer }}-R_{\mathrm{xy}}^{\text {outer }}}{\left(R_{\mathrm{xy}}^{\text {outer }}\right)^{2}},
$$

where $R_{\mathrm{xy}}^{\text {outer }}=3 h / e^{2}$ and $R_{\text {Diag }}^{\text {outer }}=\left(1 / R_{\text {Diag }}-e^{2} / h\right)^{-1}$, thus subtracting the conductance of the $v=1$ edge channel. Figure 6(b) shows $g_{\text {tun }}^{\text {outer }}$ calculated from the same data as $g_{\text {tun }}$ in Fig. 6(a). At the lowest temperatures, the value of $g_{\text {tun }}^{\text {outer }}$ at zero bias is strongly affected by measurement noise (as $g_{\text {tun }}^{\text {outer }}$ diverges for $R_{\text {Diag }} \rightarrow 1$ ) and thus cannot be analyzed, but for temperatures higher or equal to $17.8 \mathrm{mK}$, we obtain a temperature dependence compatible with a power law. The width and temperature dependence of the zero-bias maximum in $g_{\text {tun }}^{\text {outer }}$ are roughly similar to the curve described by Eq. (2) assuming $g=1 / 3$ and $e^{*}=e / 3$ [black crosses in Fig. 6(b)]. In contrast, for the tunneling conductance $g_{\text {tun }}$ calculated from raw data, the decrease of the peak's height with increasing temperature is slower than predicted by Eq. (2) [black crosses in Fig. 6(a)].

The overall shape of the $g_{\text {tun }}^{\text {outer }}$ curve obtained by evaluating Eq. (3) is not compatible with the weak backscattering model, in particular at high values of dc bias, preventing a systematic least-square fitting analysis. We can thus not make a statement 
about the best fit parameters $e^{*}$ and $g$. We do however find that with respect to Wen's model with the values of $e^{*} / e$ and $g$ set to $1 / 3$, the temperature dependence of the zero-bias peak in $g_{\text {tun }}^{\text {outer }}$ shows better qualitative agreement than the temperature dependence of the zero-bias peak in $g_{\text {tun }}$. We thus propose that subtracting the conductance of the perfectly transmitted $v=1$ mode is a step in the right direction in the theoretical treatment of QP tunneling in the $v=3 / 4$ state.

\section{DISCUSSION AND CONCLUSION}

We have measured the tunneling conductance between edge states of the fractional quantum Hall effect at $v=1 / 3$ and $v=4 / 3$ and have compared our data quantitatively to the model derived by Wen within a perturbative treatment of the chiral Luttinger liquid [3]. For both states, in the case of low backscattering we observe a bias dependence of the tunneling conductance which cannot be explained by currently available theories.

At filling $v=1 / 3$, our data is qualitatively compatible with the model only in the limit of high backscattering. This result is puzzling, because the model is valid only for low tunneling amplitudes. Furthermore, in the case of high backscattering charge carrier density is significantly reduced in the QPC and backscattering might be mediated by tunneling of single electrons instead of quasiparticles. Nevertheless, the best fit is obtained with an effective charge in quantitative agreement with the predicted value $e^{*}=e / 3$. We observe that the extracted interaction parameter $g$ depends on gate voltage, in agreement with the prediction that $g$ is affected by geometric effects in typical split Hall bar geometries [28].

Our results at filling $v=4 / 3$ indicate that the edge supports an $v=1 / 3$ mode and a $v=1$ mode, and that only the $v=1 / 3$ mode contributes to the tunneling conductance across the QPC. For higher backscattering strengths, if we account for the perfect transmission of the $v=1$ mode and consider the tunneling conductance of the outer $v=1 / 3$ mode, we obtain a temperature dependence of the zero-bias maximum in rough agreement with Wen's model assuming the scaling parameters $e^{*}=e / 3$ and $g=1 / 3$ predicted for a $v=1 / 3$ edge mode. The overall shape of the bias dependence cannot however be accounted for. Here, a more involved theoretical treatment of our experiment relying on the exact solution [23] for the tunneling conductance might bring more clarity.

\section{ACKNOWLEDGMENTS}

The authors wish to thank Dmitri Feldman for useful discussions and gratefully acknowledge the support of the ETH FIRST laboratory and the financial support of the Swiss Science Foundation (Schweizerischer Nationalfonds, NCCR QSIT).
[1] A. M. Chang, Rev. Mod. Phys. 75, 1449 (2003).

[2] F. P. Milliken, C. P. Umbach, and R. A. Webb, Solid State Commun. 97, 309 (1996).

[3] X. G. Wen, Phys. Rev. B 44, 5708 (1991).

[4] X. G. Wen, Phys. Rev. B 43, 11025 (1991).

[5] X. G. Wen, Phys. Rev. Lett. 64, 2206 (1990).

[6] I. P. Radu, J. B. Miller, C. M. Marcus, M. A. Kastner, L. N. Pfeiffer, and K. W. West, Science 320, 899 (2008).

[7] X. Lin, C. Dillard, M. A. Kastner, L. N. Pfeiffer, and K. W. West, Phys. Rev. B 85, 165321 (2012).

[8] S. Baer, C. Rössler, T. Ihn, K. Ensslin, C. Reichl, and W. Wegscheider, Phys. Rev. B 90, 075403 (2014).

[9] H. Fu, P. Wang, P. Shan, L. Xiong, L. N. Pfeiffer, K. West, M. A. Kastner, and X. Lin, Proc. Natl. Acad. Sci. USA 113, 12386 (2016).

[10] R. B. Laughlin, Phys. Rev. Lett. 50, 1395 (1983).

[11] R. B. Laughlin, Surf. Sci. 142, 163 (1984).

[12] R. de Picciotto, M. Reznikov, M. Heiblum, V. Umansky, G. Bunin, and D. Mahalu, Nature (London) 389, 162 (1997).

[13] L. Saminadayar, D. C. Glattli, Y. Jin, and B. Etienne, Phys. Rev. Lett. 79, 2526 (1997).

[14] S. Roddaro, V. Pellegrini, F. Beltram, G. Biasiol, L. Sorba, R. Raimondi, and G. Vignale, Phys. Rev. Lett. 90, 046805 (2003).

[15] S. Roddaro, V. Pellegrini, F. Beltram, G. Biasiol, and L. Sorba, Phys. Rev. Lett. 93, 046801 (2004).
[16] S. Roddaro, V. Pellegrini, and F. Beltram, Solid State Commun. 131, 565 (2004).

[17] S. Roddaro, V. Pellegrini, F. Beltram, G. Biasiol, L. Sorba, R. D’Agosta, R. Raimondi, and G. Vignale, Physica E 22, 185 (2004).

[18] S. Roddaro, V. Pellegrini, F. Beltram, L. N. Pfeiffer, and K. W. West, Phys. Rev. Lett. 95, 156804 (2005).

[19] S. Roddaro, N. Paradiso, V. Pellegrini, G. Biasiol, L. Sorba, and F. Beltram, Phys. Rev. Lett. 103, 016802 (2009).

[20] A. Stern and B. I. Halperin, Phys. Rev. Lett. 96, 016802 (2006).

[21] Magnicon GmbH, "Mfft-1 product description and datasheet" (2018).

[22] B. J. Overbosch, Edge tunneling and transport in non-abelian fractional quantum Hall systems, Ph.D. thesis, Massachusetts Institute of Technology, 2008, http://hdl.handle.net/1721.1/45163.

[23] P. Fendley, A. W. W. Ludwig, and H. Saleur, Phys. Rev. B 52, 8934 (1995).

[24] P. Fendley, A. W. W. Ludwig, and H. Saleur, Phys. Rev. Lett. 74, 3005 (1995).

[25] T. G. Griffiths, E. Comforti, M. Heiblum, A. Stern, and V. Umansky, Phys. Rev. Lett. 85, 3918 (2000).

[26] M. Heiblum, Phys. Status Solidi B 243, 3604 (2006).

[27] S. Hennel, B. A. Braem, S. Baer, L. Tiemann, P. Sohi, D. Wehrli, A. Hofmann, C. Reichl, W. Wegscheider, C. Rössler, T. Ihn, K. Ensslin, M. S. Rudner, and B. Rosenow, Phys. Rev. Lett. 116, 136804 (2016).

[28] E. Papa and A. H. MacDonald, Phys. Rev. B 72, 045324 (2005). 